
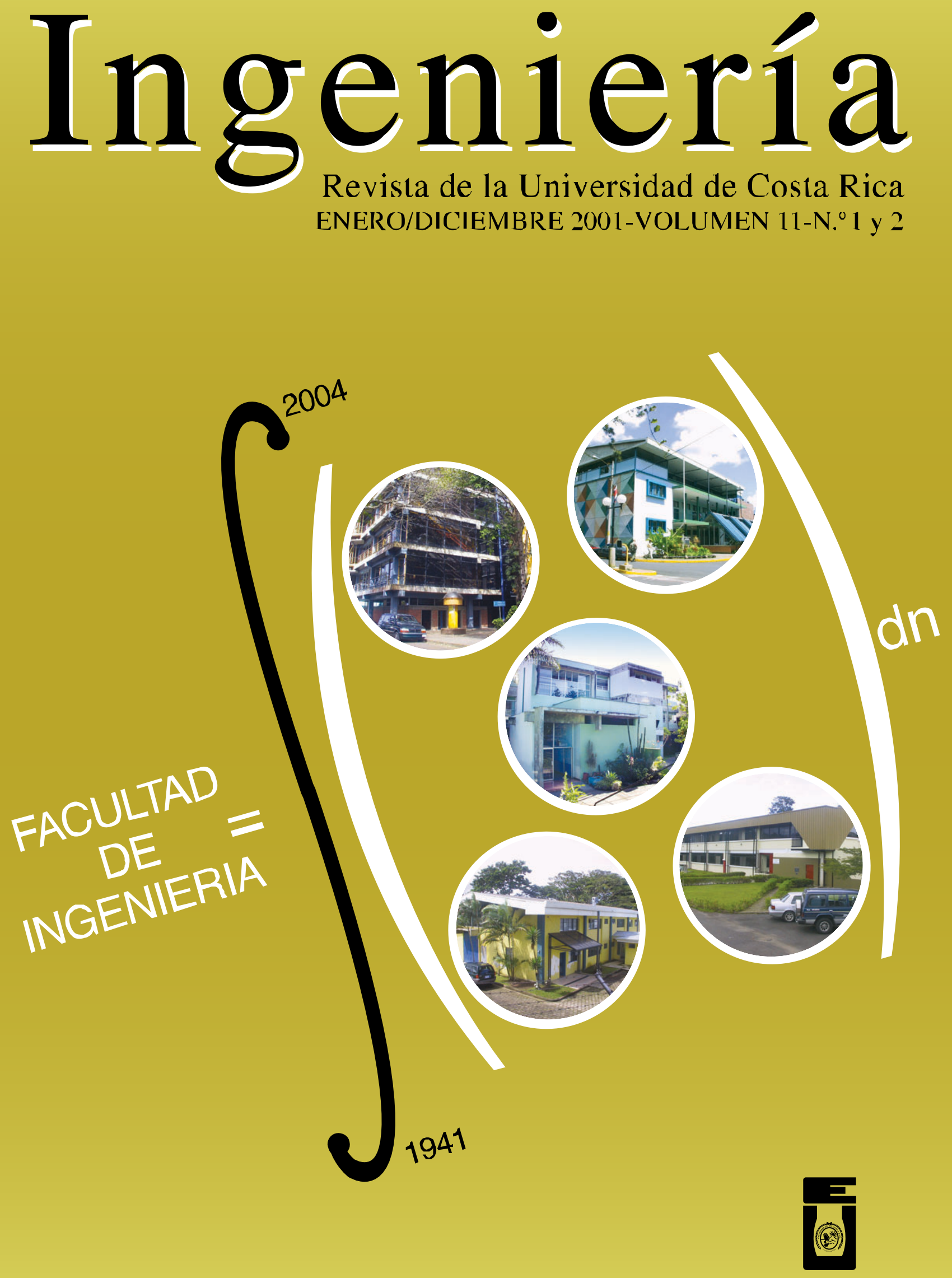
Comentarios 



\title{
DON FERNANDO CARBONI E. UNA SEMBLANZA: IN MEMORIAM
}

\author{
Ing. Manuel A. Calvo $H$. \\ Ing. Rodolfo Herrera J.
}

\begin{abstract}
Resumen
Don Fernando Carboni E. fue una figura importante en la vida cultural e intelectual de la Universidad de Costa Rica en el ámbito de la Ingeniería, la Física y las Matemáticas. Su labor en esas disciplinas es desconocida para las nuevas generaciones de académicos y profesionales. La breve semblanza que presentamos tiene el propósito de dar a conocer su intensa y profunda vida intelectual, dedicada a la enseñanza y la investigación.

Don Fernando Carboni E. dejó de existir el 11 de setiembre del 2001 y su muerte ha dejado un gran vacío en el corazón de sus amigos y compañeros de estudio y de trabajo, especialmente en los autores de esta semblanza.
\end{abstract}

\begin{abstract}
Mr. Fernando Carboni E. played an important role for the cultural and intellectual life of the Costa Rica University in the engineering, physics and mathematics fields. His labor is unknown for the new generations of academics and professionals. This brief portrait has the purpose to vindicate his intense and deep intellectual life dedicated to teaching and researching about the costarrican culture.

Mr. Fernado Carboni E. expired September 11, 2001 and his dead left a deep emptiness in the heart of his friends, classmates and colleagues, in particular the authors of this portrait.
\end{abstract}

Este distinguido ingeniero civil fue el primer costarricense en obtener un doctorado en Física (Ph.D), en la Universidad de Kansas, Estados Unidos. Nació en Cartago el 10 de diciembre de 1929. Comenzó sus estudios de Ingeniería Civil en la Universidad de Costa Rica en 1948 y se graduó en 1956 , al presentar su magnífica tesis Bóvedas cáscara hiperbólico-parabólicas de concreto armado.

Carboni fue el primer ingeniero costarricense en incursionar en la Ingeniería Estructural al desarrollar un método para obtener las ecuaciones que resuelven el problema de la estática para paraboloides hiperbólicos, utilizando una superficie tórica (toro o toroidal), que sirve como modelo de las superficies de doble curvatura, con características tales, que en un punto cualquiera posea los mismos radios de curvatura que la superficie dada y con las secciones principales coincidentes. De ese modo habrá un contacto de segundo orden entre ambas super- ficies y las ecuaciones diferenciales, que representan el fenómeno, serán idénticas. Fue un trabajo de carácter teórico, inusual en el país para aquella época. Con este trabajo, Don Fernando daba los primeros pasos de una vida que estaría dedicada a la investigación y la enseñanza de la ciencia.

En 1950 los autores éramos estudiantes de Ingeniería Civil y reafirmamos la amistad con Fernando, quien cursaba el tercer año de la carrera. Mantuvimos durante años una relación intelectual y cultural marcada por un mutuo interés en la ciencia. En ese entonces no existía en el país una carrera universitaria de Física o Matemáticas, así que su estudio era autodidáctico. El interés del profesor Carboni, así como el nuestro, por los campos físico-matemáticos, se incrementó con las enseñanzas del profesor Luis González G. (1 905 - 1 962), que a la sazón era en Costa Rica la persona que tenía más conocimientos matemáticos y con quien 
mantuvimos una gran amistad, compartiendo y discutiendo ideas propias del quehacer académico, específicamente en campos tan nuevos en el país como el análisis tensorial o la teoría de la relatividad.

En esa época, Don Fernando participó con el Doctor Gil Chaverri R., el ingeniero Enrique Soto B. (QDG) y los autores, en actividades y encuentros relacionados con la necesidad de crear una carrera de Física y Matemáticas. Se organizaron algunos cursos especiales de matemáticas para todos aquellos que estuvieran interesados, como el de Álgebra Moderna y Álgebra Lineal, dictados por el Ms. matemático y profesor José Joaquín Trejos F. expresidente de la república-; un curso de Geometría Descriptiva, dictado por el profesor Luis González, y otros más.

Junto con el profesor Carboni, los autores y otros colegas y profesores vinculados alrededor de Luis González G., - los ingenieros Walter Sagot C. (QDG), Fernando Chavarría L(QDG), Enrique Soto B(QDG) y el doctor Gil Chaverri R.-, constituyeron un núcleo de estudiantes y profesores que sirvió como base y elemento de cultivo intelectual para la fundación de la Escuela de Física y Matemáticas de la Universidad de Costa Rica en 1 957, la cual contó desde el principio con otros excelentes académicos llegados de la enseñanza secundaria nacional como los ingenieros Elliot Cohen P. y Henry McGhie, los profesores de matemática Juan Félix Martínez y Bernardo Alfaro -primer director de la Escuela de Física y Matemática, el profesor de física Fabio González, el profesor José Joaquín Trejos, así como algunos profesores extranjeros.

Una vez graduado de ingeniero civil, Don Fernando realizó muchos trabajos en el campo de la Ingeniería, en especial en la Ingeniería Estructural. Inició su carrera de profesor universitario en el año 1 957, año de la llamada "reforma universitaria" en la Universidad de Costa Rica; primero como profesor de Álgebra y Trigonometría, y a partir de 1960 comenzó a impartir cursos de Física. En la nueva Facultad de
Ciencias y Letras dictó el curso de Fundamentos de Matemáticas, coordinado por el catedrático español Dr. Roberto Saumells, experto en Filosofía de la Ciencia.

Debido a su inteligencia y capacidad mostrada en el campo de las ciencias Físico-Matemáticas, el profesor Dr. Gil Chaverri, entonces decano de la Facultad de Ciencias y Letras de la Universidad de Costa Rica, le consiguió en el año 1962 una beca para realizar estudios superiores en Física en la Universidad de Kansas, EEUU.

Su desempeño como estudiante en la Universidad de Kansas fue brillante, y obtuvo la maestría en Física en 1 964. Su tesis de graduación sirvió de base para el artículo Nuclear Alignment of $\mathrm{Co}^{60}$ in Cerium Magnesium Nitrate (1). Siguió con sus estudios para conseguir el título de doctor (Ph.D) en Física en la Universidad de Kansas, y lo logró en el año de 1967 con la presentación de su sobresaliente tesis: Resonancia Magnética en Cadenas de Heisenberg (2). También en 1 967, junto con ilustres físicos norteamericanos, publicó: Exchange and the "10/3 effect" in $\mathrm{Cu}\left(\mathrm{NH}_{3}\right)_{4} \mathrm{SO}_{4} \cdot \mathrm{H}_{2} \mathrm{O}$, (3); y Observations and calculation of the "10/3 effect" in $\mathrm{Cu}\left(\mathrm{NH}_{3}\right)_{4} \mathrm{SO}_{4} \cdot \mathrm{H}_{2} \mathrm{O}$ confirm a non-Gaussian form for one - dimensional spin time correlation functions (4).

A fines de 1967 regresó a nuestro país. Trabajó en el antiguo Departamento de Física y Matemática, y luego partió hacia la República de Venezuela donde trabajó durante diez años, dos en la Universidad de Cumana y ocho en la Universidad Metropolitana de Caracas; en la primera se dedicó a la docencia y a la investigación en Física, y fue director del Laboratorio de Mecánica de Suelos; en la segunda, además de dedicarse a la docencia, fue director del Departamento de Física. De la Universidad de Caracas salió la publicación: Spin Waves in Finite Spin-1/2 Heisenberg Chains* (5).

Hemos mostrado apenas unas pocas publicaciones de Don Fernando; sin embargo, el número de artículos sobre investigaciones en física del estado 
sólido y otros tópicos publicados en revistas de mucho prestigio internacional es enorme.

Poseía un sinnúmero de cualidades humanas entre las que destacan su buen trato con todos los que fueron compañeros, amigos y subalternos; así como su humildad, sensibilidad, amor a las artes y al estudio, y sobre todo el espíritu de solidaridad que demostraba en el momento de brindar ayuda o consuelo, tanto es así que pese a su árduo trabajo en la tesis doctoral en la Universidad de Kansas, 1 966, respondió a la necesidad de un amigo, el Ing. Calvo, quien sufriera la pérdida de su hija menor tras una enfermedad cardíaca que la llevó a la muerte. Se dedicó por entero a prestarle atención al amigo y a su familia para que pudieran sobrellevar la gran crisis emocional, que conlleva una tragedia de esta envergadura. De aquí el agradecimiento eterno de su amigo y compañero.

En su juventud practicó algunas veces béisbol, pero sobre todo se apasionó por el ajedrez y en la década del 50 formó el equipo de la Universidad de Costa Rica, junto con los ingenieros civiles Ricardo Charpentier S. y Rodolfo Silva V y llegó a participar en varios campeonatos nacionales. Fue una persona sumamente amable, le gustaba participar en toda clase de reuniones y disfrutaba mucho compartiendo con sus amigos temas diversos. Meses antes de su muerte le acompañamos a vistar en su lecho de enfermo a nuestro compañero y amigo de interesantes tertulias intelectuales, el ingeniero y economista Enrique Soto Borbón (Qdg).

Hasta aquí hemos hecho un análisis de la personalidad de Don Fernando y hemos intentado describir sólo un pequeño resumen de sus años pasados en Estados Unidos y Venezuela. Regresó a Costa Rica en 1979, año a partir del cual empezó a trabajar en Docencia e Investigación en la Escuela de Física de la Universidad de Costa Rica. Simultáneamente, fue profesor en universidades privadas de nuestro país, impartiendo cursos en la carrera de Ingeniería Civil, principalmente los relativos a Estructuras Elementales y Estructuras Avanzadas. Se jubiló en el año 1989.
Entre sus trabajos de ingeniería más importantes se encuentran los siguientes diseños estructurales: la Parroquia del Carmen de Cartago, el Edificio del Banco Central de Costa Rica, el edificio de la Corte Suprema de Justicia de San José, los puentes para la empresa consultora Consultécnica, edificios de apartamentos en Caracas, Venezuela y un sinnúmero de estructuras civiles. En los últimos 10 años trabajó con uno de los suscritos, el Ing. Manuel Calvo H., en el diseño e inspección de otra gran cantidad de obras.

También fue autor de un programa de computación en el lenguaje Basic y aplicable en D.O.S., para el diseño de estructuras tridimensionales sometidas a cualquier clase de cargas, lo llamó ELFI y es un programa que usa la teoría del elemento finito al análisis de estructuras.

No queremos terminar este pequeño resumen de la vida del profesor Fernando Carboni sin antes indicar, como ya se describió en párrafos anteriores, que él fue uno de los precursores de lo que hoy en día es la prestigiosa Escuela de Física y Matemática de la Universidad de Costa Rica. Fue un científico de altos quilates, de labor académica silenciosa pero profunda, dedicado al conocimiento de las ciencias, que cultivó con amor y una seriedad admirable. Apegado al pensamiento racional y a la ética científica, fue un ejemplo para sus amigos, alumnos y compañeros. Su desaparición, tal vez muy temprana, ha sido una irreparable pérdida para el mundo intelectual del país.

\section{REFERENCIAS}

1. Fernando Carboni and Richard C. Sapp, of Department of Physics and Astronomy, the University of Kansas, Lawrence, Kansas, 1 965. Reprinted from Annals of Physics, Volume 33, $\mathrm{N}^{\circ} 1$, June 1965 . Copyright by Academic Press Inc. Printed in U.S.A. Annals of Physics: , 77-87 (1 965).

2. Este trabajo de investigación fue publicado como artículo bajo el nombre de Time Dependence of Spin Operators in Finite Heisenberg Linear Chains* por Fernando 
Carboni and Peter M. Richards, Department of Physics, University of Kansas, Laurence Kansas 66044 (Received July 12, 1968). Reprinted from the Physical Review, Vol. 177, No. 2, 889_904, January 10, 1969.

3. Robert N. Rogers, Sandia Laboratory, Albuquerque, New Mexico.

4. Fernando Carboni and Peter M. Richards,
Department of Physics, University of Kansas, Lawrence Kansas.

5. Physical Review B., Volume 5, Number 5, 1 march 1972, by Peter M. Richards, Department of Physics, University of Kansas, Lawrence, Kansas 66044 and Fernando Carboni, Department of Physics, Universidad de Oriente, Cumana, Venezuela (Received June 2, 1 971). 